\title{
Deconvolution of hardness from data obtained from nanoindentation of rough surfaces
}

\author{
M.S. Bobji and S. K. Biswas ${ }^{\mathrm{a}}$ \\ Department of Mechanical Engineering, Indian Institute of Science, Bangalore, 560 012, India
}

(Received 2 October 1998; accepted 15 March 1999)

\begin{abstract}
Variation of hardness with penetration in nanoindentation of a rough surface is a compound effect of variation in asperity geometry with penetration, designated geometric effect, and genuine property gradients with depth as may exist in a near-surface zone. We simulate indentation of a rough surface numerically to elucidate the geometric effects and validate it by some model "macro" experiments. Finally, we formulate a general framework to deconvolute genuine property variation by normalizing the measured hardness with the geometric effect.
\end{abstract}

\section{INTRODUCTION}

When there is a relative motion between bodies in contact, power is dissipated. For example, this occurs in manufacturing of parts as in machining and metal working as well as in machineries of locomotion, power generation, and machine tools. The regions of contact in this situation are severely stressed and give rise to acute gradients of strain, strain rate, and temperature. The large strains near the surface change the topography, through flow or fracture, while the combination of strain rate and temperature in the near surface zone may change microstructure to promote cracking or flow. What precisely is the material response depends on how the microstructure evolves. So phenomenologically the topography and microstructure and therefore the topography and surface mechanical properties stem from the same process, the response of a material to traction.

To an engineer both the topography and surface mechanical properties are interesting as they contribute not only to the loss of power through friction but also to the life cycle of the active components. The measurement of topography is now possible over a wide range of length scales, down to atomic scales using atomic force microscopy (AFM). The indentation technique is generally used to probe mechanical properties of solids, ${ }^{1,2}$ and the nanoindenter is being used now to probe surface properties where the scale of probing is comparable to that of the topography. It has been found that at low penetration depths, the hardness is different from the bulk hardness and the scatter in the measurement is high. ${ }^{3}$ Pollock et al. ${ }^{4}$ have reviewed the relevant theory and the experimentation that describe the behavior of materials in the 10-1000 nm depth range.

\footnotetext{
a)Address all correspondence to this author.

e-mail: skbis@mecheng.iisc.ernet.in
}

The variation in hardness at low penetration depths may be attributed to surface chemical effects, ${ }^{3,5}$ material property variation with depth, ${ }^{3}$ and/or surface roughness of the specimen being indented. The variation is also influenced by the method of measurement: depth sensing or imaging and by the instrument errors in depth-sensing measurement. The errors associated with nanoindentation measurement have been discussed by Menčik and Swain, ${ }^{6}$ and the scatter has been studied by Yost. ${ }^{7}$ Here we are concerned with the effect of roughness on the surface mechanical property estimates made using nanoindentation.

It is not difficult to visualize as Tabor ${ }^{8}$ had noted many years ago that the effect of roughness on hardness estimation is negligible if the indentation depths are much greater than the surface roughness. The self-affine fractal nature of engineering surfaces has been demonstrated since then using scanning tunneling microscopy (STM) and AFM. ${ }^{9}$ The roughness wavelengths that affect a physical process are determined by the length scale of the process. For nanoindentation the appropriate length scale is the size of the indent made. The effect of asperities much smaller than the indent size is averaged out, as for example in the case of conventional hardness measurements. Similarly the asperities that are much larger than the indent do not affect the measurements as they present almost a plane surface to the indenter.

We have studied machined, ground, and polished metal surfaces using standard profilometer as well as AFM to find the surface power spectra to be much influenced by material properties at $1 \mathrm{~mm}$ to $10 \mathrm{~nm}$ length scales though the slopes of the spectra are independent of material properties at the submicronic scale. ${ }^{10}$ Further, the power spectra obtained by polishing a (aluminum) surface was found to be a virtual extension of the spectra obtained by grinding the same surface. This means that while polishing may reduce the amplitude of roughness at length scales greater than a certain value 
determined by the abrasive grit size, at the submicronic scale it continues the same topographical architecture as imprinted by coarser removal processes such as grinding and machining. Thus except in the case of cleaved, atomistically smooth surfaces, nanoindentations are invariably and effectively carried out on rough surfaces.

Interpretation and analysis of nanohardness data obtained from engineering surfaces pose several challenges.

1. The mean pressure in any contact ${ }^{11}$ being related to the geometry of contact, the topography even in the absence of any real property gradient affects the mean pressure. Visualizing a rough surface as made up of asperities of small radius riding on the back of asperities of larger radius, ${ }^{12}$ penetration by indenter of any geometry brings asperities of small radius into play first (Fig. 1). With increasing penetration, asperities of larger radius are encountered. As the effective radius encountered by the indenter tip continues to change with penetration, the strain varies and thus the measured hardness has to change with penetration. When nanoindentation is carried out on a rough surface, with no property gradient, the hardness estimates are thus invariably dependent on the penetration depth.

2. If there is a genuine mechanical property gradient such that the property is related, for example, to the volume of deformed material, the measured hardness independent of the above geometric effect relates to the depth of indentation.

3. The topography itself is the result of large strains and specific local strain rate responses. An attempt to deconvolute the "flat surface" property gradient by normalizing the measured hardness with the geometric effect can lead to error especially if the acute strain rate and temperature gradients promote discontinuities and unstable material responses such as adiabatic shear banding or wedge cracking at the asperity level. If the indentation was done on a "prepared" perfectly smooth surface, such responses may be absent.

4. Asperities by the very nature of their formation are repositories of high residual stresses and strain ener-

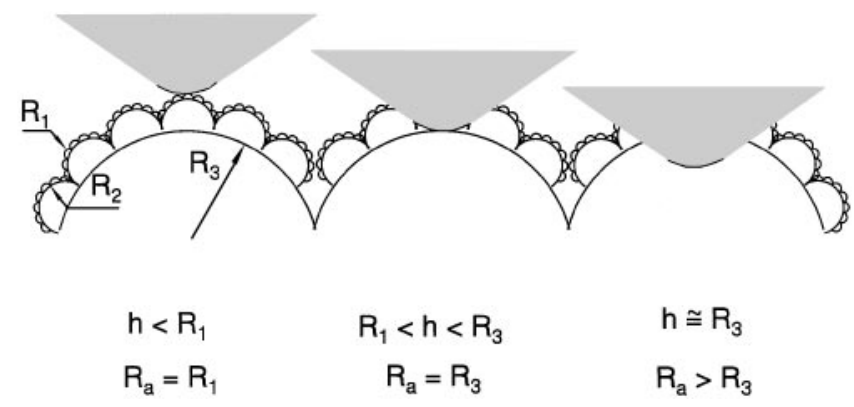

FIG. 1. Schematic of an indenter on a fractal surface. As the penetration $(h)$ of the indenter increases, the effective asperity radius $\left(R_{a}\right)$ increases. gies. It is possible that the intervention by the indenter may give rise to fracture even at the lightest of loads. The deconvolution of nanohardness data by taking into account the disturbance produced by the observer's probe may be a difficult problem to circumvent.

Given the quantitative description of the surface roughness, we are concerned here to develop a method to deconvolute flat surface hardness of a material from nanohardness measurements done on a rough engineering surface. In our work we assume that there is no discontinuity in property gradient with depth. In other words, we discount the possibility that the variation of property with depth is dependent on the location of measurement, as long as the measurement commences from a free surface irrespective of whether that free surface belong to an asperity or a prepared flat surface. We further ignore any possible disruptive effect of the indenter on the hardness measurement. We pursue our program by first establishing the purely "geometric effect." This is done by performing macroindentation of a soft spherical body by a rigid spherical indenter. The experiment gives us an indication of hardness dependence on penetration in a single asperity contact. Using these results we next simulate the indentation of a fractal surface by a rigid spherical indenter by taking into account the contact area and mean pressure prevailing at each contacting asperity. In establishing a general framework for deconvolution of surface hardness based on our simulation data, we assume an error term ${ }^{13}$ to be directly proportional to rms roughness. We next investigate this proportionality by conducting indentation experiments on a specially fabricated rough surface consisting of equispaced pyramidal asperities. Having found the proportionality to be linear and the proportionality constant being independent of asperity geometry, we validate the general framework for the special case rough surface and proceed with our simulation of the indentation of a general rough surface which is fractal. The simulation is carried out for surfaces with and without property gradients with depth. We finally arrive at an equation where direct substitution of rms roughness yields the flat surface hardness at a depth, given the measured hardness.

\section{SINGLE ASPERITY CONTACT}

When an indenter is brought to a rough surface, the contact is established at a single asperity level. If the radius of the asperity is very small compared to that of the indenter, then the response is as that of a rigid flat surface pressed against the asperity. As the indenter is displaced further, the radius of the asperity increases and becomes comparable to the tip radius of the indenter (Fig. 1). The contact configuration at this state can be approximated to that of a rigid sphere indenting a hemisphere, as shown in Fig. 2. The variation of the 

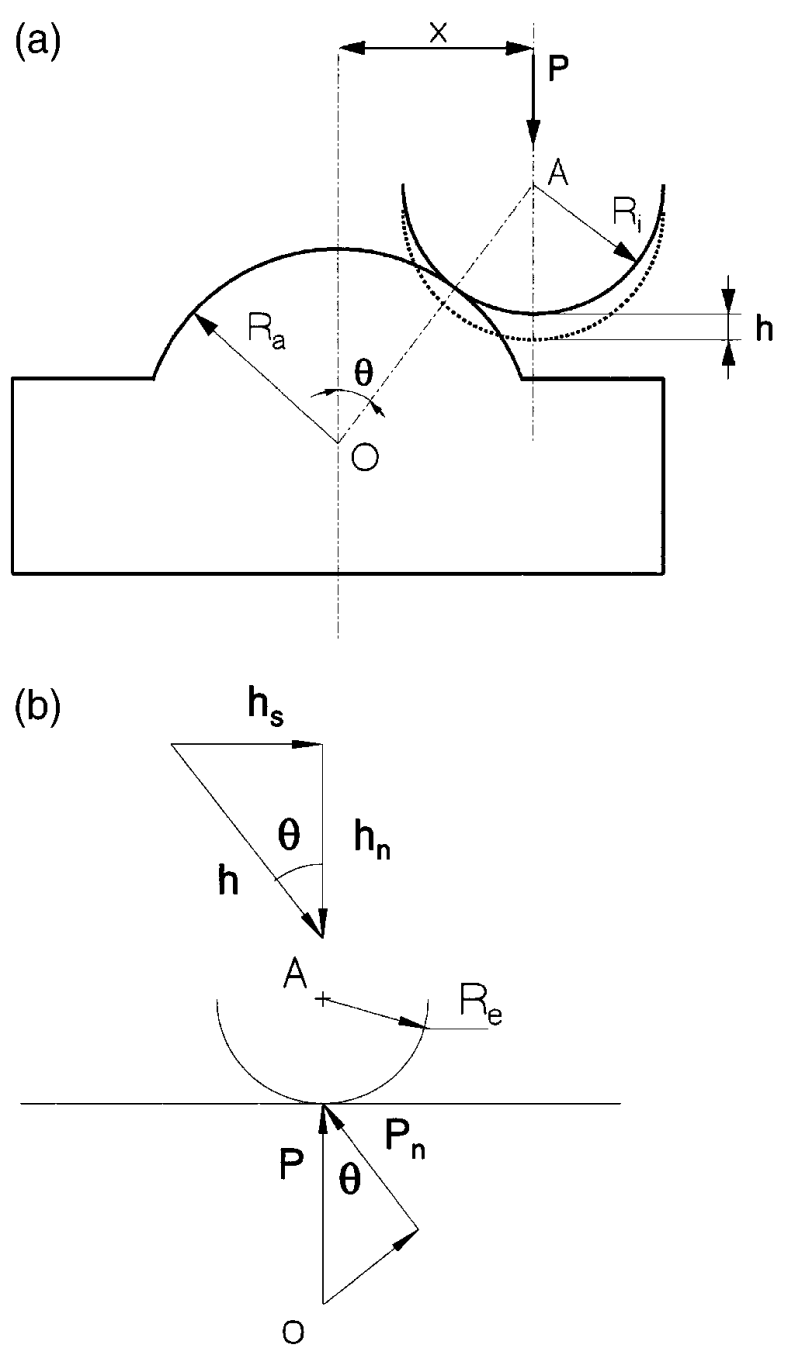

FIG. 2. Single asperity contact: (a) configuration and (b) sum surface.

measured hardness will depend on (i) the radius of the asperity $\left(R_{a}\right)$, (ii) the radius of the indenter $\left(R_{i}\right)$, and (iii) the relative position of the indenter and the asperity (x) that determines the angle $\theta$.

The single asperity contact, where the indenter and the asperity radii are comparable, is studied by macroscopic indentation experiments in which hemispherical copper asperities are indented with hard steel balls of radius $\left(R_{i}\right) 12.5 \mathrm{~mm}$. The scale of the experiment is selected such that the effect of the variation in the strength of the material with the deformation volume is negligible. Specimens as shown in Fig. 2(a), and of three different radii $\left(R_{a}\right)$, i.e., $25,12.5$, and $8 \mathrm{~mm}$, were machined out of copper rods in a copying lathe. A fixture was used to position the specimen such that the distance between the specimen and indenter axes (x) can be varied. Indentation was carried out in a 10 ton $(\approx 100 \mathrm{KN})$ universal testing machine, and loadpenetration curves were recorded. ${ }^{14}$ The experimental material stiffness $(=50 \mathrm{KN} / \mathrm{m})$ was found to be two orders less the machine stiffness of $6 \mathrm{GN} / \mathrm{m}$.

A typical load-penetration depth curve obtained from the experiment is shown in Fig. 3. The hardness is measured by drawing a tangent to the unloading portion of the curve and taking the $y$-intercept of this tangent for calculating the area of residual impression. ${ }^{1}$ However, from Fig. 3 it can be seen that the initial portion of the unloading path is nearly normal to the penetration axis. Hence, the maximum penetration depth is used here to calculate the hardness. The difference between the maximum penetration $(4.4 \mathrm{~mm})$ at $80 \mathrm{KN}$ load and the residual penetration at no load was found to be $0.04 \mathrm{~mm}$. This was considered to be small and therefore neglected. The hardness (the ratio of applied load to projected area) is calculated as,

$$
H=\frac{P}{A f(h)}=\frac{P}{\pi\left(2 R_{i} h-h^{2}\right)},
$$

where $A f(h)$ is the area function of the indenter. Indentation experiments were carried out on a flat specimen and the hardness estimated from these experiments is taken as $H_{s}$.

The sum surface of the contact ${ }^{15}$ will be a hemisphere [Fig. 2(b)] with a reduced radius,

$$
R_{e}=\frac{R_{a} R_{i}}{R_{a}+R_{i}}
$$

If the indenter is displaced by $h$ along its axis, then the penetration along the line OA will be $h \cos \theta$. The

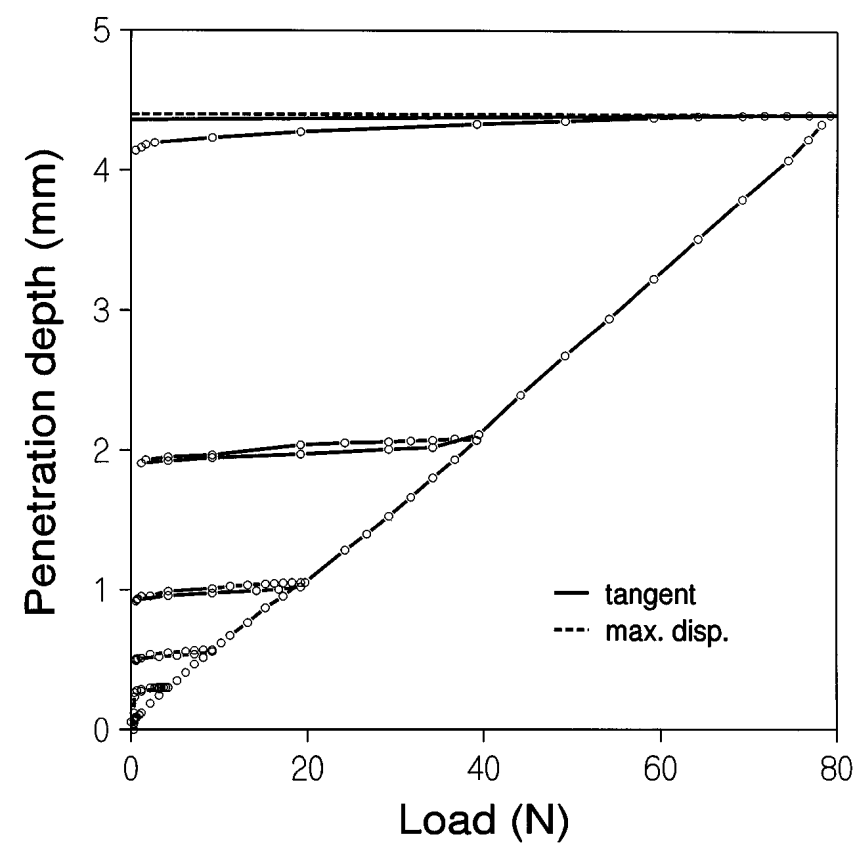

FIG. 3. Load-penetration depth characteristics of single asperity contact, $R_{a}=12.5 \mathrm{~mm}$ and $R_{i}=12.5 \mathrm{~mm}$. 
normal load along OA,

$$
P_{n}=H_{o} 2 \pi R_{e} h \cos \theta
$$

where $H_{o}$ is the mean pressure, which can be obtained by indenting a smooth flat surface. Resolving this load along the axis of the indenter, the measured hardness [Eq. (1)] can be written as,

$$
H=H_{o}\left(\frac{R_{a} \cos ^{2} \theta}{R_{i}+R_{a}}\right)
$$

Thus the measured hardness can be expressed as a product of a geometry factor and the material property $\left(H_{o}\right)$. Figure 4 shows the experimental data collected at different angular offsets $(\theta)$ and specimen radii $\left(R_{a}\right)$ to fall roughly on a single straight line when plotted as a function of $\left(R_{a} \cos ^{2} \theta\right) /\left(R_{i}+R_{a}\right)$.

From Eq. (2) it can be seen that the hardness measured is less than the bulk hardness obtained on a flat surface. Further, when the asperity radius increases (as it does with the increasing penetration of the indenter), the measured hardness approaches the bulk value asymptotically.

When the asperity radius is large compared to the indenter radius in the later stage of single asperity indentation, Eq. (2) can be written by expanding the term within the parentheses and neglecting the higher order terms of $R_{i} / R_{a}$ as,

$$
\frac{H}{H_{o}} \approx 1-\frac{R_{i}}{R_{a}}
$$

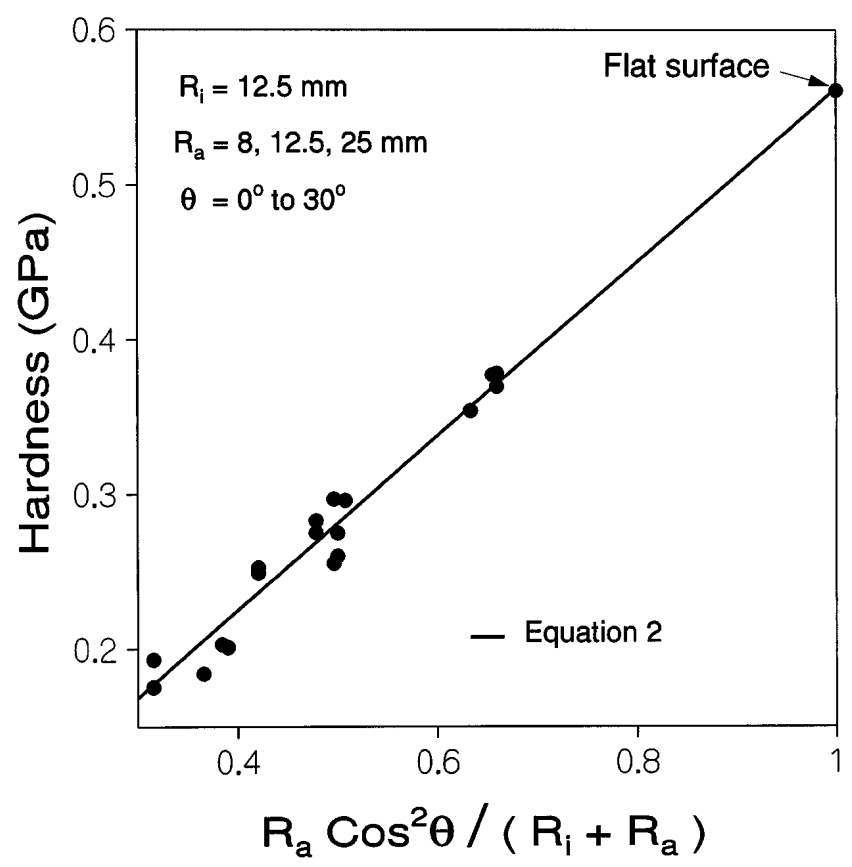

FIG. 4. Plot of hardness versus $R_{a} \cos ^{2} \theta /\left(R_{i}+R_{a}\right)$. for $\cos \theta \approx 1$. The values of $\theta$ are restricted to small values to avoid multiple asperity contact. $R_{a}$ varies continuously for an actual rough surface from zero to infinity as the indenter penetrates into the surface.

When an indenter makes contact with a rough surface the contact initially (stage I) occurs with a single asperity. In stage II more than one asperity makes contact with the indenter, the actual contact area being a sum of many tiny contact islands. The measured hardness is determined by the distribution of these contact islands, the distribution being related to the statistical character of the rough surface. We can now conceptually formulate an equivalent asperity whose radius $\left(R_{a}\right)$ is a function of the radii of all the current asperities in contact. Clearly this radius is a function of the current penetration depth of the indenter and the statistical nature of the surface roughness. When the penetration of the indenter is further increased to stage III, then a contact island of size considerably greater than the surrounding tiny islands emerges (inset of Fig. 8). This has been verified experimentally for pyramidal asperities.

The equivalent asperity radius $\left(R_{a}\right)$ may be written in a general form as

$$
R_{a}=K_{1}\left(\frac{h}{h_{r}}\right)^{m},
$$

where $h_{r}$ is a roughness parameter, which characterizes the statistical nature of the rough surface such as root mean square roughness, and $K_{1}$ and $m$ are the constants. Equation (3) may now be combined with Eq. (4) to give,

$$
\frac{H}{H_{o}}=1-\frac{K_{2}}{\left(\frac{h}{h_{r}}\right)^{m}},
$$

where $K_{2}=R_{i} / K_{1}$.

\section{MULTIPLE ASPERITY CONTACT}

We focus our attention on stage III of the indentation of a rough surface. While we formulate a general framework for deconvolution of hardness from the indentation data obtained from a rough surface, we experimentally validate the framework for a special case where the surface contains equispaced identical pyramidal asperities. The validation leads to the formulation of the general framework which is applied to deconvolute hardness from the indentation of a fractal surface with and without a property gradient with depth.

\section{A. Experimental}

Square pyramidal asperities of different apical angles were machined on a specimen (Fig. 5) of diameter $50 \mathrm{~mm}$ made of a commercially pure work-hardened copper rod. 


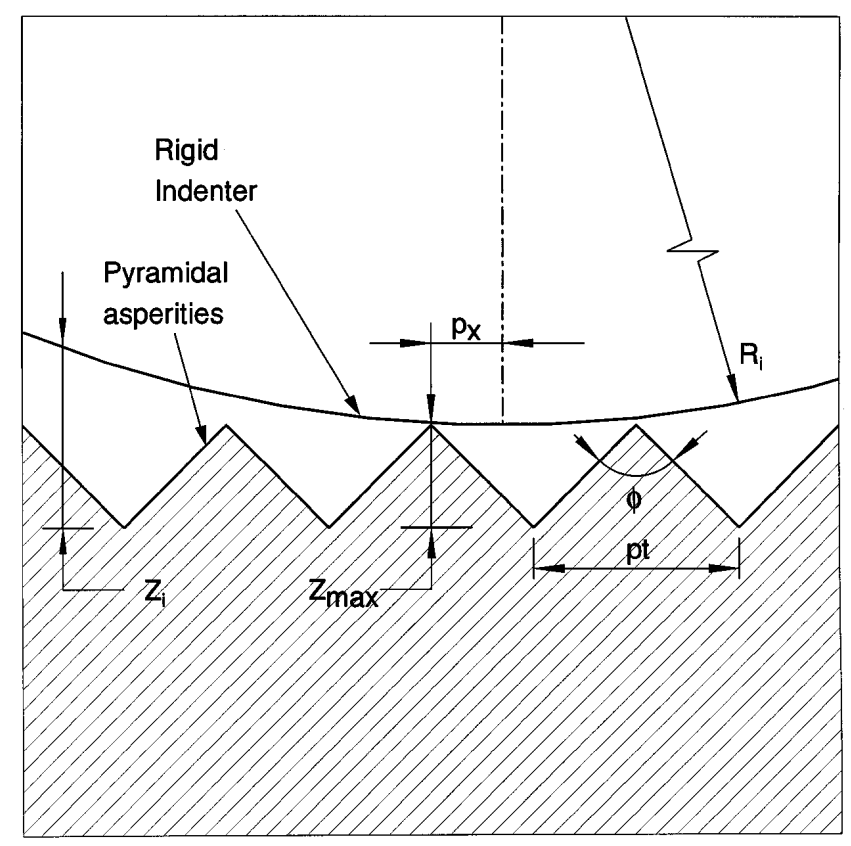

FIG. 5. Schematic of the pyramidal asperity contact.

The pyramids were generated in a shaping machine using a V-shaped HSS tool. The angle of the tool is varied to get pyramids with three different angles $(\phi)$ between the faces: $45^{\circ}, 60^{\circ}$, and $90^{\circ}$. First, V-shaped grooves are generated on the specimen by moving the tool with a feed equal to the pitch $(p t)$. Then the specimen is indexed through $90^{\circ}$ and machined with the same feed and depth of cut. The depth of cut given is slightly more than the height of the pyramid $\left(Z_{\max }\right)$ to ensure a sharp apex. The roughness of the specimen could be varied by varying the apical angle $(\phi)$ of the pyramids as well as the pitch $(p t)$. If $Z$ is the height of the asperity above the base plane, then the root mean square roughness is given by, ${ }^{16}$

$$
\begin{aligned}
h_{r} & =\frac{1}{\sqrt{18}} Z_{\max } \\
& =\frac{1}{\sqrt{18}} \frac{p t}{2 \tan \phi} .
\end{aligned}
$$

A total of 15 specimens were machined with three different angles $(\phi)$ and five different pitches for every angle. Indentations were carried out using a hard spherical indenter of diameter $25 \mathrm{~mm}$ in a 10 ton universal testing machine using the same setup as the single asperity experiments. Figure 6 shows a typical experimental load-penetration depth characteristics with the different stages of deformation marked.

Weiss ${ }^{17}$ pointed out that the effect of roughness on the measured hardness $\left(H_{r}\right)$ on a rough surface can be accounted for by adding an error term $h_{e}$ in penetration depth. This would give ${ }^{14}$

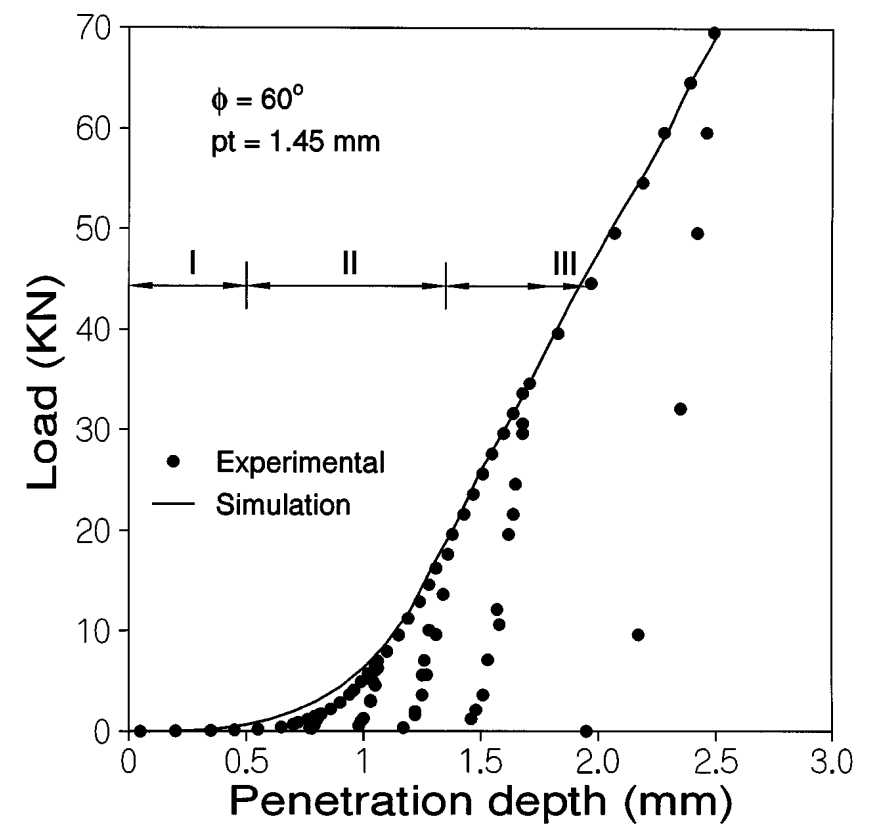

FIG. 6. Comparison between the experimental and simulated load-penetration depth characteristics of pyramidal asperities.

$$
\begin{aligned}
\frac{H_{r}}{H_{o}} & =\frac{A f\left(h \pm h_{e}\right)}{A f(h)} \\
& =\left(1 \pm \frac{h_{e}}{h}\right) \quad \text { for spherical } \\
& =\left(1 \pm \frac{h_{e}}{h}\right)^{2} \quad \text { for conical/pyramidal },
\end{aligned}
$$

where $H_{o}$ is the bulk hardness and $A f$ is the area function of the indenter. Comparing this with Eq. (5) it is clear that for the assumed dependency of effective radius on penetration [Eq. (4)], $h_{e}$ is related to some roughness parameter and the indenter geometry. Accordingly we may write

$$
h_{e}=k h_{r}
$$

and

$$
\frac{H_{r}}{H_{o}}=\left(1-\frac{k}{h / h_{r}}\right)^{n}
$$

where $k$ and $n$ are parameters dependent on indenter geometry; $n$ for Weiss's analysis is 1 for a spherical indenter and 2 for a conical indenter.

In Fig. 7, the experimentally obtained hardness $(H)$ normalized with the hardness measured on a smooth flat surface $\left(H_{o}\right)$ at the same penetration $h$ is plotted against the penetration normalized with the rms roughness $\left(h / h_{r}\right)$. Equation (7) is plotted as the continuous line, with $n=1$ for spherical indenter and $h_{e}=3.4 h_{r}{ }^{16}$ It can be seen that all the experimentally measured hardnesses fall, irrespective of asperity geometry and 


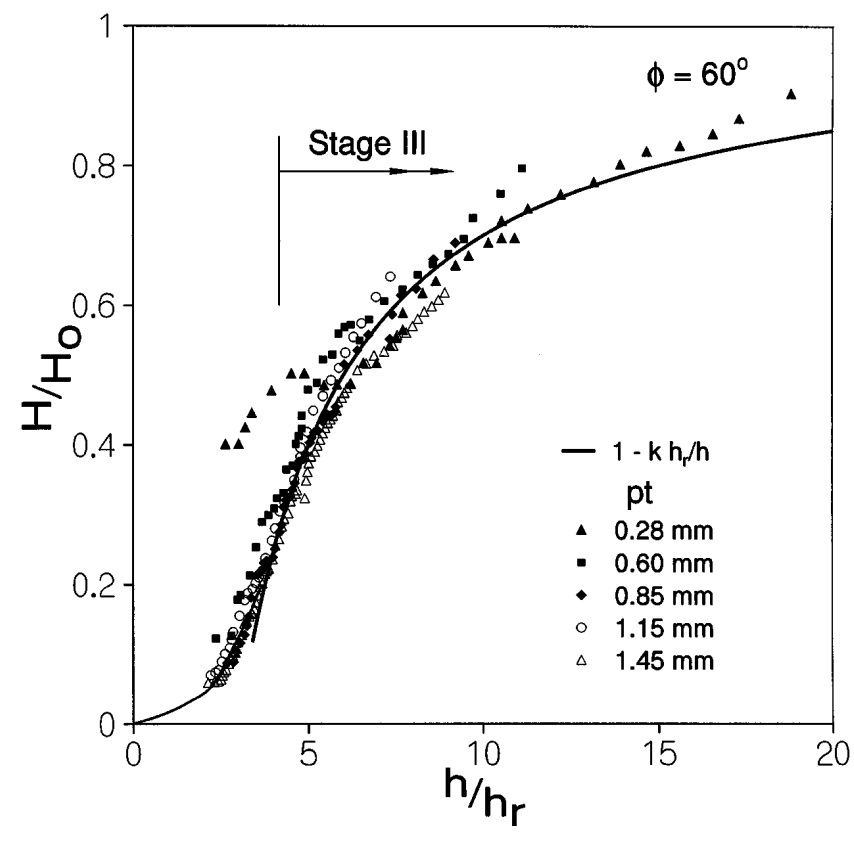

FIG. 7. Variation of the normalized hardness with normalized penetration for pyramidal asperities for different asperity pitches $(p t)$.

spacing, on this line which asymptotically approaches the flat surface hardness $\left[\left(H / H_{o}\right)=1\right]$ at large penetrations. The similarity in trend and levels between the expected and actual values of measured hardness validates the assumption made regarding the nature of the error term $h_{e}$.

\section{B. Numerical simulation}

For the numerical simulation the pyramidal surface is generated over a $256 \times 256$ grid. The smooth spherical indenter, which is also generated over the same grid, is brought into contact with the simulated surface and the contact area is obtained. For this, first the sum surface ${ }^{15}$ is found using

$$
Z_{s}=Z_{i}-Z
$$

$Z_{s}$ gives the difference in height between the indenter $Z_{i}$ and the rough surface $Z$ (Fig. 5). The contact area for a particular penetration $(h)$ of the indenter into the rough surface is the contour of the sum surface for the value of $Z_{s}$ equal to $h$. The contours were obtained using a standard algorithm that uses linear interpolation for the $Z_{s}$ values in between the grid points.

The indentation of a soft rough surface by a smooth and hard indenter is equivalent to the penetration of a soft smooth and flat surface by a set of hard asperities. ${ }^{8}$ From the volume and the area of a contact island, the spherical cavity model ${ }^{11}$ is used to estimate the mean pressure acting over the contact island.

$$
p_{i}=\frac{2}{3} Y\left\{2+\left[\ln \frac{\frac{E \tan \beta}{2 Y}+2(1-2 \nu)}{3(1-\nu)}\right]\right\},
$$

where $E$ is Young's modulus, $Y$ is the yield strength, and $\nu$ is Poisson's ratio of the material being indented. $\tan \beta$ is obtained by equating the volume of the contact island to that of a cone of attack angle $\beta$ whose base area is equal to the contact area of the island. It is assumed that the indenter deformation is negligible. The upper limit to this mean pressure is set by the condition of fully plastic deformation. Thus the load supported by each island is computed as

$$
P_{i}=\left\{\begin{array}{ll}
p_{i} \times A_{i} & \text { if } p_{i}<3 \\
3 \times Y \times A_{i} & \text { if } p_{i} \geqslant 3
\end{array},\right.
$$

where $A_{i}$ is the contact area of the individual islands. The total load is obtained by summing up the individual load supported by all the islands for a given penetration.

In Fig. 6 the simulated load $(P)$ versus penetration depth $(h)$ characteristics is compared with that obtained experimentally. It can be seen that the match between the simulation and the experimental characteristics is very good for penetrations greater than $1 \mathrm{~mm}$. At penetrations less than $1 \mathrm{~mm}$ the yield strength $(\approx H / 3)$ of the copper is less than $0.2 \mathrm{GPa}$, as can be seen from the flat surface hardness variation in Fig. 6. As a constant yield strength of $0.2 \mathrm{GPa}$ is assumed in simulation, the load is slightly higher than that obtained from experiment. It can also be noted that the stage III characteristics are the same as that for the sphere on a flat surface shifted along the penetration depth axis by a constant $h_{e}$.

\section{INDENTATION ON A FRACTAL SURFACE}

To study the effect of the variation of the strength with the size of the asperities, we carry out a simulation study on a rough surface. The rough surface is simulated by a fractal function in order to consider the roughness in the wavelength scales of less than the resolution of the profilometers $(\approx 1 \mu \mathrm{m})$. The height variation $Z(x)$ of an isotropic and homogeneous rough surface in any arbitrary direction, along a straight line, can be represented by the Weierstrass-Mandelbrot function. ${ }^{18}$

$$
\begin{gathered}
Z(x)=G^{(D-1)} \sum_{n=n_{l}}^{\infty} \frac{\cos \left(2 \pi \gamma^{n} x\right)}{\gamma^{(2-D) n}} \\
1<D<2 ; \quad \gamma>1 ;
\end{gathered}
$$

In this $G$ is a scaling constant, $D$ is the fractal dimension of the profile, $\gamma^{n}=1 / \lambda$ is the frequency mode corresponding to the reciprocal of the wavelength $(\lambda)$ of roughness, $\gamma^{n_{l}}$ is the lower cut-off frequency of the profile which depends on the length of the sample $L$ through the relation $\gamma^{n_{l}}=1 / L$, and $\gamma$ is chosen to be 1.5 for phase randomization and high spectral density.

A value of 1.5 is chosen for $D$, corresponding to brownian surface. ${ }^{9}$ The summation to infinity is cut off at a high index. The indices are chosen to be 34 and 
52 , respectively, such that the roughness is simulated in the same length scale as the physical phenomena-the indentation to be studied. The surface is simulated over a grid of $128 \times 128$ uniformly spaced points. ${ }^{13,19}$

The geometry of the indenter used is shown in Fig. 8. The half cone angle $(\alpha)$ and the tip radius of curvature $\left(R_{i}\right)$ are varied to get the different area function. The surface of the indenter $Z_{i}$ is generated over the same set of grid points as the simulated rough surface. The axis of the indenter is varied randomly over the $x-y$ plane, within the $1 \times 1 \mu \mathrm{m}$ simulated surface. Twentyfive such random indentations are carried out for given indenter parameters $\alpha$ and $R_{i}$.

The hardness is obtained from Eq. (1). The penetration, $h$, used to obtain the area through the area function can be measured from two different reference planes. One is the plane passing through the initial contact point of the indenter with the rough surface and parallel to the mean plane. This simulates a depth-sensing indentation experiment with ideally infinite measurement resolution. The other plane is the mean plane of the rough surface. This simulates the imaging type of nanoindentation experiments. ${ }^{20}$ The area function for a given indenter geometry is computed using the same routine but by letting the indentation be done on a smooth flat surface.

The indentation was carried out at 25 random locations on the simulated surface, and the load was found out for 11 different penetration depths at a given location. The rms roughness $\left(R_{\mathrm{rms}}\right)$ of the indented surface is varied by varying the magnification constant

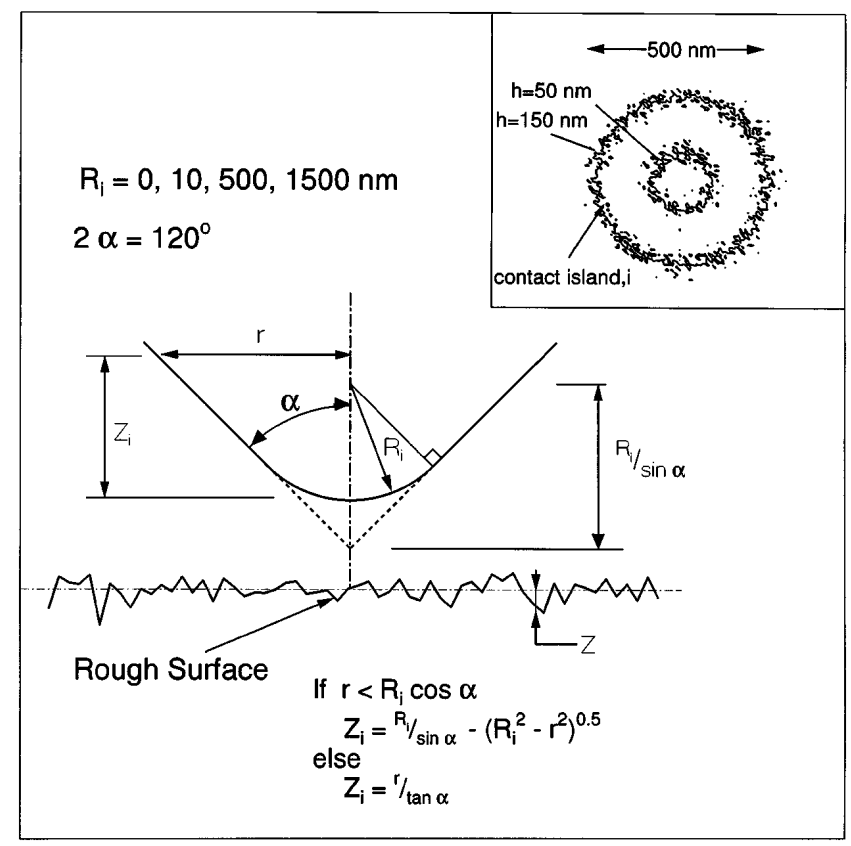

FIG. 8. Schematic of the fractal surface contact used in simulation. Inset shows the actual contact area for two different penetrations of the indenter.
$G$ in Eq. (4). ${ }^{9}$ The results are summarized in Fig. 9. The figure clearly brings out the fact that even for a material the bulk and the surface mechanical properties of which are the same, the hardness changes with penetration as long as the surface is rough. The figure further delineates the influence of the method of measurement and the actual property gradient with depth on the measured hardness.

To introduce the effect of the varying material property with the deformation volume or the penetration depth, the yield strength $Y$ used in Eq. (8) to calculate the mean pressure is allowed to vary as

$$
Y=Y_{o}\left(1+\frac{c_{0}}{V^{n}}\right) \text {. }
$$

With $n=1 / 3$ this would give, for a conical indenter, a flat surface hardness variation of type,

$$
H=H_{o}\left(1+\frac{c_{1}}{h}\right),
$$

where $H_{o}$ is the bulk hardness and $c_{1}$ is a material constant. Figure 10 shows the spread of the estimated hardness points normalized with the rough surface hardness [Eq. (7)] as a function of the penetration depth and roughness, for a sharp conical indenter.

For a general material with an arbitrary property variation, the hardness measured on a flat smooth surface can be written as

$$
H=H_{o} f(h) .
$$

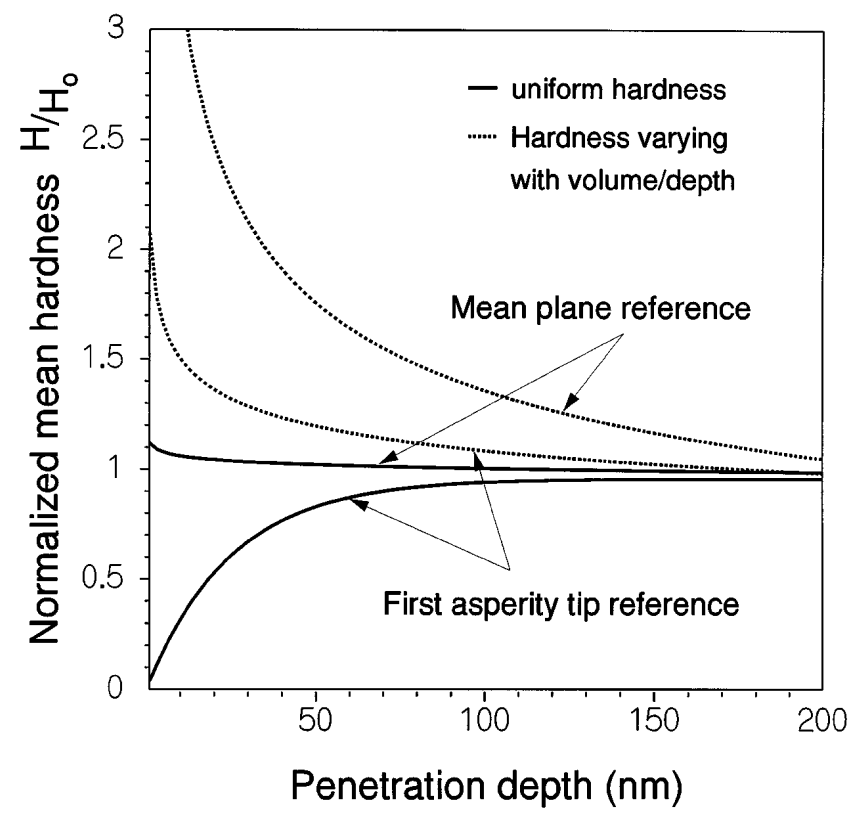

FIG. 9. Summary of the mean hardness variation with penetration for a conical indenter with a tip radius of $10 \mathrm{~nm}$ indenting a fractal surface. 


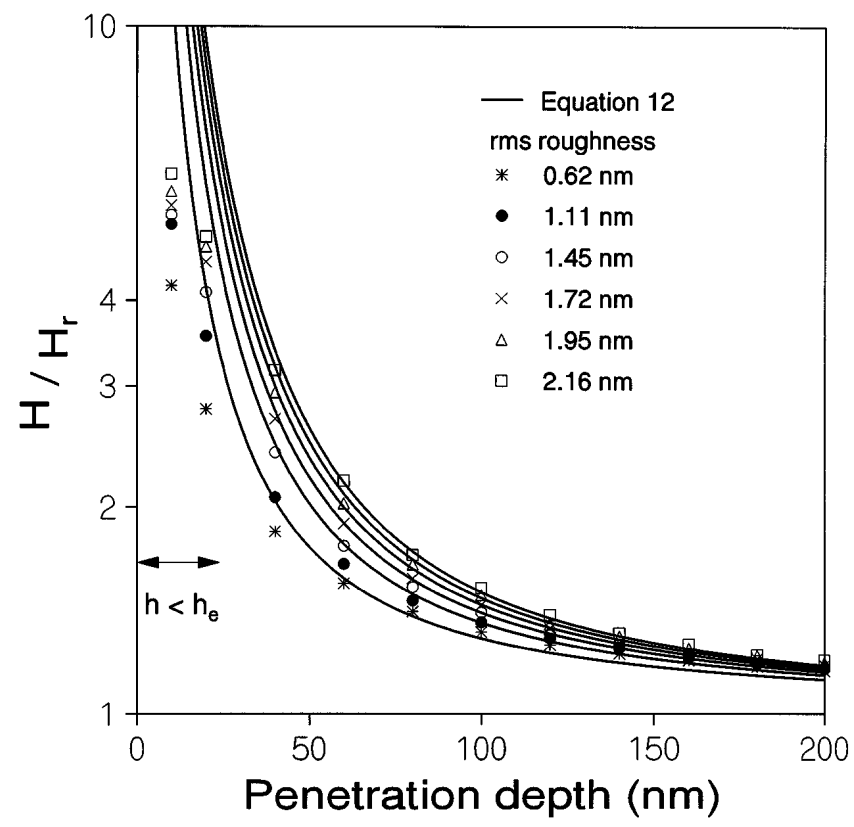

FIG. 10. Variation of the mean hardness normalized with the hardness measured on a rough surface with no property gradient, for a conical indenter with zero tip radius for different surface roughnesses.

Since the effect of roughness could be accounted for by adding an error term to penetration,,$^{13,17}$ when a rough surface of such a material is indented, the hardness measured could be obtained as,

$$
H=H_{r} f\left(h-h_{e}\right) .
$$

Thus, the arbitrary function can be obtained from the hardness $(H)$ measured in a nanoindentation experiment by plotting $H / H_{r}$ against $h-h_{e}$. Knowing the bulk hardness $\left(H_{o}\right), H_{r}$ can be obtained from Eq. (7); $h_{e}$ can be obtained from Eq. (6). The values $k$ and $n$ have been obtained from numerical simulation for different indenters on a fractal surface. ${ }^{13}$

In the numerical simulation, a property variation of the type given by Eq. (10) was assumed. Thus Eq. (11) becomes,

$$
H=H_{r}\left(1+\frac{c_{1}}{h-h_{e}}\right)
$$

Substituting for $h_{e}$ from Eq. (6), this can be written in a series form for $h>h_{e}$ as

$$
H=H_{r}\left(1+\frac{c_{1}}{h}+\frac{c_{1} k h_{r}}{h^{2}}+\frac{c_{1}\left(k h_{r}\right)^{2}}{h^{3}}+\cdots\right) \text {. }
$$

The continuous lines in Fig. 10 are drawn as per the above equation, limiting the series to the first 3 terms. It can be seen that the variation of hardness due to the changing roughness and penetration depth is described well by this equation. Substitution for $H_{r}$ from Eq. (7), the hardness measured on a rough surface with a material property variation can be written as

$$
H=\left(1-\frac{k}{h / h_{r}}\right)^{n}\left(1+\frac{c_{1}}{h}+\frac{c_{1} k h_{r}}{h^{2}}\right) H_{o} .
$$

The first term in this equation gives the hardness variation due to roughness alone when there is no property variation with volume/depth. The second term expresses the effect of property gradient in its interaction with roughness on hardness. This term comes about because the deformation volume in an asperity, for a given penetration depth, changes with roughness. This results in a change in the aggregate strength of the asperity. The roughness thus alters the asperity-wise distribution of strength and geometric constraint. Hardness, which is a product of strength and constraint summed over the whole contact domain, changes with roughness. When there is no property variation with volume $c_{1}$ is zero and the hardness reduces to Eq. (7). When the roughness, on the other hand, is zero $\left(h_{r}=0\right)$, Eq. (13) reduces to the smooth surface material property profile [Eq. (10)].

\section{v. CONCLUSIONS}

To test the efficacy of the method developed, we conducted nanoindentation experiments on a mechanically polished surface. Work-hardened copper (99.9\% pure) was indented in a displacement controlled instrument ${ }^{21}$ with piezo drive and capacitive sensors. The force is measured as a deflection of an elastic hinge. The copper surface was mechanically polished with a diamond paste of average diamond size of $2.5 \mu \mathrm{m}$. The rms value of such polished surface is $7.5 \mathrm{~nm}$. This is obtained from a profile measured using an atomic force microscope over a sampling length of $10 \mu \mathrm{m}$ with a sampling interval of $25 \mathrm{~nm}$. The conical diamond indenter has a tip radius $R_{i}$ of about $30 \mu \mathrm{m}$. Figure 11 shows the variation of the measured hardness normalized with bulk hardness $\left(H_{o}\right)$.

Taking the values of $k$ and $n$ from the numerical simulation, ${ }^{13} H_{r}$ can now be written for this indenter as [Eq. (7)]

$$
H_{r}=H_{o}\left(1-\frac{25}{h / h_{r}}\right) .
$$

Substituting the values of Young's modulus ( $E=$ $123 \mathrm{GPa})$ and Poisson's ratio $(\nu=0.33)$, the function $f(h)$ can be obtained.

In Fig. 12, the $H / H_{r}$ is plotted against $h-h_{e}$ and gives the corrected hardness variation with penetration. The continuous line is the best fit of equation of type,

$$
\frac{H}{H_{o}}=1+\frac{c_{1}}{\left(h-h_{e}\right)^{m}} .
$$

The index $m$ is very close to 1 , which gives the variation of type given by Eq. (10) assumed for the numerical 


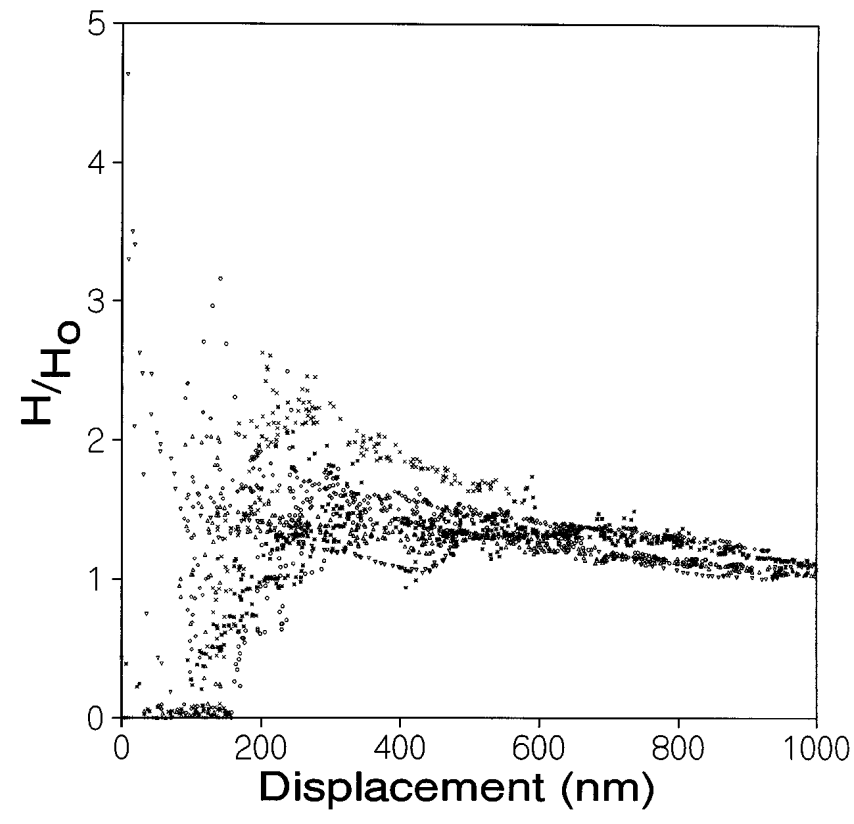

FIG. 11. Hardness normalized with the bulk hardness obtained by nanoindentation of a mechanically polished copper surface. The various symbols refer to different sets of experiments.

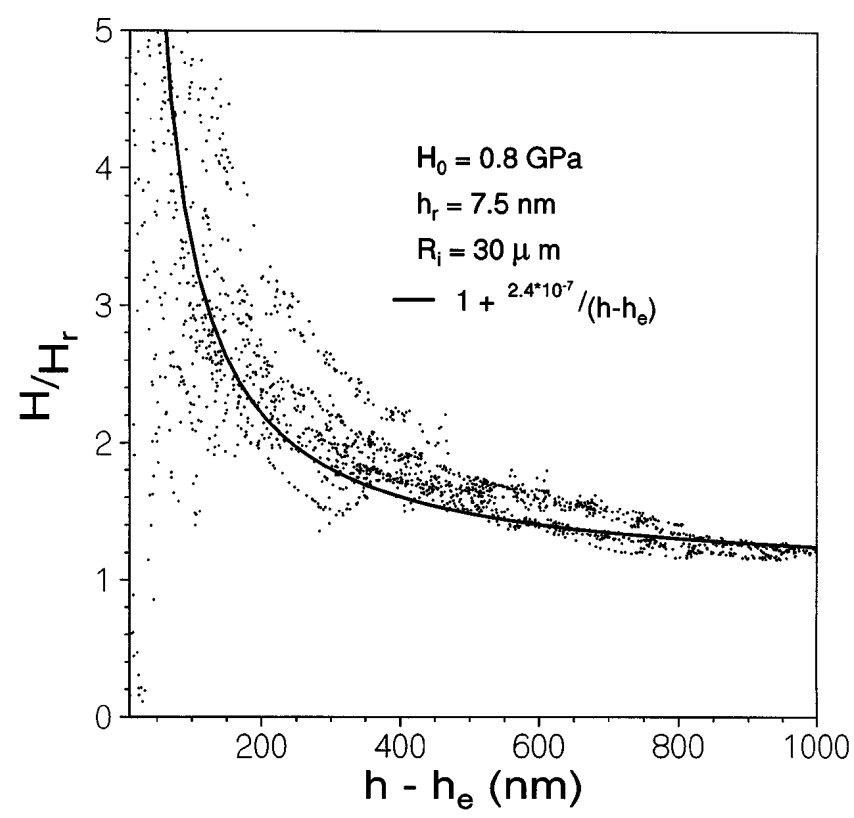

FIG. 12. Nanoindentation data corrected for the effect of rough surface.

simulation. Comparing Fig. 11 with Fig. 12, it can be seen that the correction decreases the scatter in hardness at penetration depths less than $200 \mathrm{~nm}$ and yields a hardness characteristic of $h^{-1}$ dependency found by others ${ }^{3}$ by nanoindentation of smooth flat surfaces.

For penetration depths larger than 3 times the rms roughness of a surface, the present work offers a relationship that may be used to find the genuine property variation with depth from a surface. This is, of course, valid for cases where there is no discontinuity in such variation as well as when no additional roughness is created in the process of intervention by the indenter. The strength of the proposed method, in spite of the interactive nature of the material property gradient and the roughness, is its ability to separate the geometric from the material property effect such that given the roughness the deconvolution will give the genuine property variation. The method, however, uses a geometric function that is validated here experimentally for a special case of roughness geometry and also when the penetration depth is more than 3 times the rms roughness. (For the nanoindentation data presented, this comes to about $20 \mathrm{~nm}$.)

While the limited nanoindentation data we have presented here suggest a more universal applicability of the relation in terms of surface geometry, clearly experimental work needs to be undertaken for a variety of surface architecture to test the general validity of this relationship. The deconvolution procedure for stage I and stage II, however, poses a real challenge especially when the property variation with depth may be quite acute at distances associated with these stages. It may be expected, unlike in the case of stage III, that the initial statistical distribution of asperities geometry will play a role in determining (1) the single asperity contact in stage I and (2) the distribution of island contact areas in stage II.

The geometric factor that influences hardness will thus contain probability terms. Determination of such relations should be the focus of future numerical as well as experimental study.

\section{REFERENCES}

1. M.F. Doerner and W.D. Nix, J. Mater. Res. 1, 601 (1986).

2. J.L. Loubet, J. M. Georges, and G. Meille, in Microindentation Techniques in Materials Science and Engineering, ASTM STP 889, edited by P. J. Blau and B. R. Lawn (American Society for Testing and Materials, Philadelphia, PA, 1986), p. 72.

3. W. C. Oliver, R. Hutchings, and J. B. Pethica, in Microindentation Techniques in Materials Science and Engineering, ASTM STP 889, edited by P. J. Blau and B. R. Lawn (American Society for Testing and Materials, Philadelphia, PA, 1986), p. 90.

4. H. M. Pollock, D. Maugis, and M. Barquins, in Microindentation Techniques in Materials Science and Engineering, ASTM STP 889, edited by P. J. Blau and B. R. Lawn (American Society for Testing and Materials, Philadelphia, PA, 1986), p. 47.

5. W. W. Gerberich, S. K. Venkataramanan, H. Huang, S. E. Harrey, and D. L. Kohlstedt, Acta Metall. Mater. 43, 1569 (1995).

6. J. Menčik and M. V. Swain, J. Mater. Res. 10, 1491 (1995).

7. F. G. Yost, Metall. Trans. 14A, 947 (1983).

8. D. Tabor, The Hardness of Metals (Oxford University Press, Glasgow, 1951).

9. A. Mujamdar and B. Bhusan, Trans. of ASME, J. Tribology 112, 205 (1990).

10. K. Venaktesh, M.S. Bobji, R. Gargi, and S. K. Biswas, Proc. Conference of Wear of Materials, Atlanta, GA (1999, in press). 
11. K. L. Johnson, Contact Mechanics (Cambridge University Press, Cambridge, 1985).

12. J. F. Archard, Proc. Roy. Soc. Lond. A 243, 190 (1957).

13. M. S. Bobji and S. K. Biswas, J. Mater. Sci. 13, 11 (1998).

14. M. S. Bobji and S. K. Biswas, Tribol. Lett. 2, 381 (1996).

15. H. A. Francis, Wear 45, 221 (1977).

16. M. S. Bobji and S. K. Biswas, Tribol. Lett. (1999, in press).
17. H. J. Weiss, Phys. Status Solidi A129, 167 (1992).

18. A. Majumdar and C. L. Tien, Wear 136, 313 (1990).

19. M. S. Bobji, S. K. Biswas, and J. B. Pethica, Appl. Phys. Lett. 73, 1059 (1997).

20. B. Bhusan, V.N. Koinkar, and J-A. Ruan, Proc. Instn. Mech. Eng. J1, 208 (1994).

21. M. S. Bobji, Ph.D. Thesis, Indian Institute of Science (1997). 